\title{
Practice of Transthoracic Echocardiography by Anesthesiologists-Focused, Limited, or Comprehensive?
}

The use of cardiac ultrasound in medical practice has been well known for almost 60 years. Although primarily a domain of cardiologists, anesthesiologists got involved in the practice of diagnostic intraoperative transesophageal echocardiography (TEE) during cardiac surgery for nearly two decades. The Society of Cardiovascular Anesthesiologists (SCA) and the American Society of Anesthesiologists (ASA) have jointly published clinical practice guidelines for education and practice in perioperative transesophageal echocardiography (PTE). While anesthesiologists were busy implementing the practice of TEE in the operating room, intensivists recognized the potential of transthoracic ultrasound (TTU) for hemodynamic monitoring in an intensive care unit (ICU). Intensivists learned to obtain limited TTU windows. They utilized TTU to manage hemodynamic crisis and also during resuscitation in critically ill patients. The development of live training programs by societies (e.g., the Society of Critical Care Medicine) facilitated the implementation of TTU in their clinical practice.

Having mastered the TEE skills, it is time for anesthesiologists to look at the utility of transthoracic echocardiography (TTE) in the care of perioperative patients. As surgical patients are growing older and sicker, preoperative screening and focused use of ultrasound in hemodynamically unstable and hypoxemic postoperative patients will have a major role to play in improving patient outcomes. In this issue of the Journal of Perioperative Echocardiography, Schott et al reviewed the background, uses, and indications; recommended TTE views; and described an algorithmic approach to the evaluation of hypotensive and hypoxic patients in the perioperative period.

A recently published survey has shown that training in focused transthoracic cardiac ultrasound (FoCUS) is uncommon in anesthesiology. ${ }^{1}$ Several university programs in anesthesiology across the United States are trying to establish training programs for their faculty and residents in FoCUS. Small studies from two different sites have demonstrated the usefulness of point-ofcare ultrasound programs for anesthesiology residents and interns. ${ }^{2,3}$ In spite of the enthusiasm among current generation anesthesiologists to learn TTE, establishing clinical perioperative TTE practice faces several barriers. First, no guidelines have been published yet by any of the prominent anesthesiology societies for the practice of perioperative TTE. The Accreditation Council for Graduate Medical Education (ACGME) does not mandate any training during residency or fellowship. There are no formal examination or certification process established by the National Board of Echocardiography (NBE) for limited TTE practice by anesthesiologists or intensivists. Several small-scale studies have shown the usefulness of TTE for perioperative patients, but definitely more evidence on patient outcomes is required., ${ }^{4}$ Very few centers in the United States have successfully implemented perioperative echocardiography service. ${ }^{6,7}$ It needs a strong tireless effort by anesthesiology echocardiography leadership working with cardiologists, surgeons, hospitalists, and hospital administrators to establish such a high-utility service.

Anesthesiology-based perioperative TTE service differs from cardiology-based echocardiography service in several ways, and they should complement each other to improve overall quality of care. Perioperative TTE is performed with the aim of providing safe perioperative care for patients undergoing surgical procedures. They are done and interpreted at bedside by clinicians who have the expertise in crisis management. Comprehensive TTE is done by sonographers and read by cardiologists who have acquired extensive knowledge of cardiovascular diseases. Perioperative TTE services are available even during emergency hours but comprehensive TTE is mostly done as an elective procedure. Point-of-care diagnosis established by anesthesiologists should be referred to cardiologists for further detailed evaluation after initial goal-directed crisis management in the perioperative period. This coordinated care is vital for the existence of two echocardiography service programs with different aims and objectives.

Finally, the extent of TTE examination performed by anesthesia providers should occur at three different levels. Level 1 is focused cardiac ultrasound with pointof-care TTE examination that should be allowed only during the periods of cardiorespiratory compromise and resuscitation. Training in level 1 FoCUS should be available to all anesthesia providers and will require continuing Web-based and live education credits, simulation course, and hands-on training on volunteers. Level 2 practices should be limited to advanced echocardiographers, who are already certified to perform basic or advanced perioperative TEE. Level 2 practitioners can perform limited TTE examination for preoperative evaluation in addition 
to FoCUS examination. Level 2 practitioners will be involved in teaching FoCUS to level 1 providers. Level 2 practitioners will undergo more extensive training with requirements similar to basic TEE certification. Level 3 providers should ideally be anesthesiologists trained in cardiology. Since such personnel are limited in the field of anesthesiology, any cardiac anesthesiologist certified in advanced PTE, who will fulfill the requirements for certification in adult TTE (passed adult echocardiography examination conducted by NBE; performed and interpreted the required number of TTE examinations), can be considered as level 3 providers. Level 3 providers should be involved in training levels 1 and 2 providers, implement quality improvement programs, and conduct perioperative echocardiography research. It is imperative that both perioperative TTE education and practice grow in parallel so that the surgical patient population benefits from this initiative.

\section{REFERENCES}

1. Conlin F, Roy Connelly N, Raghunathan K, Friderici J, Schwabauer A. Focused transthoracic cardiac ultrasound: a survey of training practices. J Cardiothorac Vasc Anesth 2016 Jan;30(1):102-106.

2. Mitchell JD, Montealegre-Gallegos M, Mahmood F, Owais K, Wong V, Ferla B, Chowdhury S, Nachshon A, Doshi R, Matyal R. Multimodal perioperative ultrasound course for interns allows for enhanced acquisition and retention of skills and knowledge. A Case Rep 2015 Oct1; 5(7):119-123.

3. Ramsingh D, Rinehart J, Kain Z, Strom S, Canales C, Alexander B, Capatina A, Ma M, Le KV, Cannesson M. Impact assessment of perioperative point-of-care ultrasound training on anesthesiology residents. Anesthesiology 2015 Sep; 123(3):670-682.

4. Bøtker MT, Vang ML, Grøfte T, Sloth E, Frederiksen CA. Routine pre-operative focused ultrasonography by anesthesiologists in patients undergoing urgent surgical procedures. Acta Anaesthesiol Scand 2014 Aug;58(7):807-814.

5. Cowie B. Focused cardiovascular ultrasound performed by anesthesiologists in the perioperative period: feasible and alters patient management. J Cardiothorac Vasc Anesth 2009 Aug;23(4):450-456.

6. Manecke GR Jr, Vezina DP. Perioperative transthoracic echocardiography: "universal acid"? J Cardiothorac Vasc Anesth 2009 Aug;23(4):447-449.

7. Shillcutt SK, Brakke TR, Thomas WR, Porter TR, Lisco SJ. The development of a perioperative echocardiography consult service: the Nebraska experience. J Cardiothorac Vasc Anesth 2015;29(3):777-784.

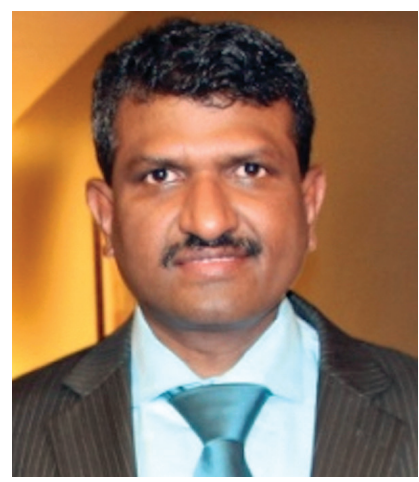

Kathirvel Subramaniam

Associate Professor, Department of Anesthesiology, University of Pittsburgh Director of Intraoperative Echocardiography, UPMC Presbyterian Hospital Pittsburgh, Pennsylvania, USA 